\title{
Synthesis and Characterization of Gold Nanoparticles - A Fuzzy Mathematical Approach
}

\author{
D. Dutta Majumder ${ }^{1}$, Sankar Karan ${ }^{2}$, and A. Goswami ${ }^{3}$ \\ ${ }^{1}$ ECSU, Indian Statistical Institute, 203, B.T. Road, Kolkata 700108, India \\ ${ }^{2}$ Institute of Radiophysics and Electronics, C.U., 92, APC Road, Kolkata 700006, India \\ ${ }^{3}$ Biological Science Division, ISI,203, B T Road, Kolkata 700108, India \\ duttamajumder.isi@gmail.com, sankar.karan@gmail.com, \\ agoswami@isical.ac.in
}

\begin{abstract}
This article presents the development of nanoparticles (NPs) with potentially useful size and shape dependent properties that have the advantage of ultra-fine size, high surface area and useful interfacial imperfections. When developing NPs as catalysts, their shape is very important. For a certain volume of material, nanoparticles make the best catalysts when they have a large surface area. It is a challenge to find the shape that has the largest surface area for its volume. The particle shape contours were measured by transmission electron microscope with high resolution. These TEM images are analyzed with image clustering techniques and generalized shape theory that results the computational indicators for shape, degree of atomic compactness and charge arrangement of NPs.
\end{abstract}

Keywords: Fuzzy C-Means Clustering, Generalized Shape Theory \& Metric, Nanomaterial, Nanoimaging, Nano Synthesis.

\section{Introduction}

Nanometer sized $\left(1 \times 10^{-9}\right.$ to $\left.100 \times 10^{-9}\right)$ particles are of today's interests because of their shape and size-dependent[1] physical properties. The chemical and physical properties of such aggregates, comprising only a few hundred atoms are in a transition region between the bulk and individual atomic or molecular properties. By understanding size and shape related changes in these systems, it is hoped that advanced new materials can be developed together with a raft of new technologies. Nanotechnology[2] may be defined as the development at the atomic, molecular and micro-molecular levels in the length and scale of approximately 1-100 nanometer range, to provide a fundamental understanding of phenomena and materials at the nanoscale and to create and use structures, devices and systems that have novel properties and functions because of their size and shape related properties. NPs with potentially useful size and shape dependent properties have the advantage of ultra fine size, high surface area, useful interfacial defects and so are extensively utilized as key components in electronics and optical 
devices, pharmaceutics, paints, coatings, superconductors, semi conductors. In fact the reproducible preparation of shape controlled particles using the popular colloid-chemical approach is difficult. Using in-process measurement techniques and particle image analysis, our research characterizes the synthesis of gold NPs improve compactness and sphericalness. This type of characterization helps the researchers in shape and size-based spectral tuning, biological labeling, toxicity studies and suggest general protocols to address these problems.

Imaging beneath the surface of a sample has always been a challenge to microscopy as they cannot be seen in the traditional sense, but that should not prevent us from visualizing the nanoworld. The main goal of Nanotechnology is to analyze and understand the properties of matter at the atomic and molecular level. Nondestructive, nanoscale characterization techniques[3] are needed to understand both synthetic and biological materials. In this paper we propose a new nanoscale imagebased characterization techniques to analyze and synthesis nanoscale images that requires algorithms to perform image analysis under extremely challenging conditions such as low signal-to-noise ratio and low resolution. To achieve this, we developed an imaging tools that are able to enhance images [4], detect objects and features [5] and analyze particle size and shape[6]. Here we present the algorithms, describe their representative methods, and conclude with several promising directions of future investigation. Nano scale photograph processing allows us to understand unique properties of matter at atomic and molecular level spanning a wide range of applications in areas such as nano-bio-medicine, nano-chip manufacturing, material sciences, nano-agri-biotechnologies and environmental toxicology[7-8].

\section{Materials and Methods}

Thiol, aspartic acid, Citrate protected gold nanoparticles of different size and shape were synthesized in the laboratory. Transmission Electron Microscopy(TEM) and spectroscopy study for Imaging and particle size distribution of Gold NPs were performed to observe the topology of the particles. These images are processed by fuzzy logic based clustering techniques. Mean diameter and compactness are measured automatically through algorithms by counting pixels belonging to every clusters. Surface(S) to Volume(V) ratio was measure considering the NPs are spherical in nature. Packing density is obtained by measuring the imperfections/defects from its segmented TEM images. Results are observed, compared with existing method and chemical measurement and manual counting.

\subsection{Nano Scale Image Data Clustering Using Fuzzy C-Means(FCM)}

TEM Image data of Gold NPs were (20nm ) classified using fuzzy C-Means clustering [9-11] algorithm to separate the particle from its background, identify the defect and also the molecular arrangement inside the particle and developed an 
iterative optimization procedure for classification. Let

$$
\mathrm{X}=\left\{X_{1}, X_{2}, \ldots,\right.
$$

$\left.X_{n}\right\}$ be a set of samples to be clustered into $c$ classes. Here we consider color as a feature for classification in RGB (red, green, blue) color space. The criterion function used for the clustering process is

$$
J(V)=\sum_{k=1}^{n} \sum_{x_{k} \in C_{1}}\left|x_{k}-v_{i}\right|^{2}
$$

Where $\mathrm{v}_{i}$, is the sample mean or the center of samples of cluster $i$, and $V=\left\{\mathrm{v}_{1}, \ldots\right.$, $\mathrm{v}_{\mathrm{c}}$ \}. To improve the similarity of the samples in each cluster, we can minimize this criterion function so that all samples are more compactly distributed around their cluster centers. Membership values ( $\mu$ 's) are assigned as per FCM algorithm.

In summary, the c-means clustering procedure consists of the following steps:

- S-1: Determine the number of clusters c.

- S-2: Partition the input samples into c clusters based on an approximation. If no rule of approximation exists, the Samples can be partitioned randomly.

- S-3: Compute the Cluster Centers

- S-4: Assign each input sample to the class of the closed cluster center.

- Repeat steps 3 and 4 until no change in $J$ can be made and the algorithm converges.

For cluster validity[11-12], we consider three types of measures : partition coefficient, partition entropy and compactness and separation validity function.

\subsection{Shape Based Image Registration of Gold NP's}

The perception of shape has been used for pattern recognition, computer vision, shape analysis[13], and image registration. Here we proposed a generalized method of shape analysis and shape based similarity measures, shape distance and shape metric to measured the NPs shape. The shape of an object can be defined as a subset $\mathrm{X}$ in $\mathrm{R}^{2}$ if (a) X is closed and bounded, (b) Interior of $X$ is non-empty and connected and (c) Closure property holds on interior of $\mathrm{X}$. This representation of shape remains invariant with respect to translation, rotation and scaling. Moreover another object $\mathrm{Y}$ in $R^{2}$ is of same shape to object $X \in R^{2}$ if it preserves translation, rotation and scaling invariance. In term of set these three transformations can be represented as

Translation : $Y=\{(x+a),(y+b): x, y \in X\}$

Rotation : $\mathrm{Y}=\{\mathrm{P} 1(\alpha) \cdot \mathrm{P} 2(\beta) \mathrm{X}\}$ where $\mathrm{P} 1 \& \mathrm{P} 2$ are rotation around $\mathrm{x}$ and $\mathrm{y}$ axes.

Scaling : $\mathrm{Y}=\{(\mathrm{kx}, \mathrm{ky}): \mathrm{x}, \mathrm{y} \in \mathrm{X}\}$

Distance $d_{1}$ between shape $X$ and $Y$ in $F$ is defined as follows:

$\mathrm{d}_{1}(\mathrm{X}, \mathrm{Y})=\mathrm{m}_{2}[(\mathrm{X}-\mathrm{Y}) \cup(\mathrm{Y}-\mathrm{X})]$ 
where $m_{2}$ is Lebesgue measure in $R^{2}$ and $d_{1}$ satisfies following rules: (i) $d_{1}(X, Y) \geq 0$, (ii) $\mathrm{d}_{1}(\mathrm{X}, \mathrm{Y})=0$ if and only if $\mathrm{X}=\mathrm{Y}$ (iii) $\mathrm{d}_{1}(\mathrm{X}, \mathrm{Y})=\mathrm{d}_{1}(\mathrm{Y}, \mathrm{X})$ and (iv) $\mathrm{d}_{1}(\mathrm{X}, \mathrm{Y})+$ $d_{1}(Y, Z) \geq d_{1}(X, Z$. We consider, two nano particles are of same shape if and only if one of the image is translation, Scaling and rotation of other.

\section{- Shape extraction and similarity measure}

To extract the feature of the boundary of the Region of Interest(ROI) it is helpful to represent the closed contour with a set of direction. The direction code may be taken among "n" selected points on the contour, which has same distance between any two consecutive points. The direction $\mathrm{d}$ makes an angle $45^{\circ}$ with direction $\mathrm{i}$, where real number $\mathrm{d} \in 1$ to 8 and $\mathrm{i}=(1,2, . .8)$. Let $\mathrm{d}_{\mathrm{m}}=\left(\mathrm{d}_{\mathrm{ij}}\right), \mathrm{j}=1$ to $\mathrm{n}$ where $\mathrm{m}=\mathrm{A}, \mathrm{B}$ are the contour starting from each reference point $A$ and $B$ and are denoted by $d_{A}$ and $d_{B}$ respectively. If $d_{2}$ is a rotation of $d_{1}$ then $d_{2}=d_{1}+\gamma$ for any real number $\gamma$. For all $j$ we can write $\mathrm{d}_{2}=\mathrm{d}_{1}+\gamma \forall \mathrm{j}$ and the distance function $\mathrm{D}$, in terms of the direction code between the contour of interest and the model is defined as:

$$
\mathrm{D}\left(\mathrm{d}_{1} \mathrm{~d}_{2}\right)=\sum_{\mathrm{J}=1}^{\mathrm{n}} \min \left(\left(\mathrm{d}_{1 \mathrm{j}} \mathrm{d}_{2 \mathrm{j}}\right), 8-\left(\mathrm{d}_{1 \mathrm{j}} \mathrm{d}_{2 \mathrm{j}}\right)\right)
$$

The normalized value of $\mathrm{D}$ is $\mathrm{D} / \mathrm{n}$ and the shape similarity measure between the two shapes is given by $\mu=1-\mathrm{D} / \mathrm{n}$, smaller value of $\mathrm{D}$ indicates higher degree of similarity [13].

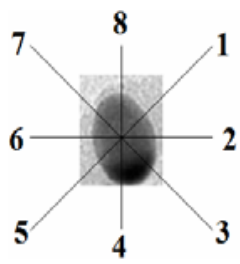

Fig. 1. Chain code representation

\section{Experimental Procedure and Results}

- Synthesis of thiol protected gold nanoparticle

Aqueous solution of $\mathrm{HAuCl}_{4}$ is mixed with solution of $\mathrm{ToABr}$ in toluene. Vigorous stirring causes transfer of $\mathrm{HAuCl}_{4}$ into the organic layer. Dodecanethiol is added to the organic phase followed by addition of aqueous solution of $\mathrm{NaBH} 4$ is slowly added with vigorous stirring. Aqueous solution of sodium borohydride is slowly added with vigorous stirring. A deep brown coloured solution appears at the interface. Excess ethanol is added to the separated brown coloured solution and kept overnight which 
causes precipitation of GNP. The ethanolic solution is filtered with nylon filter and the precipitate is re-dispersed in toluene

- Synthesis of aspartic acid protected gold nanoparticle

$90 \mathrm{ml}$ of $10^{-4} \mathrm{M}$ aqueous solution of chloroauric acid is prepared. The solution is heated up to boiling condition. $10 \mathrm{ml}$ of $10^{-2} \mathrm{M}$ aspartic acid solution is added to the boiling solution. Aspartic acid acts as the reducing agent. The reduction process is continued under constant stirring. Heating is stopped. The reduction of the metal ions is evident with appearance of red color.

\section{- Synthesis of Citrate capped gold nanoparticle}

$50 \mathrm{ml}$ of $1 \mathrm{mM}$ aqueous solution of chloroauric acid is prepared. The solution is heated up to boiling under reflux condition. $5 \mathrm{ml}$ of $1 \%$ tri sodium citrate dihydrate solution is added to the boiling solution. Sodium citrate here reduces the gold chloride. Stirring was continued until the color of the solution gradually changed from faint yellowish to clear to grey to purple to deep purple, and finally wine-red. Negatively charged citrate ions were absorbed onto the GNPs, introducing the surface charge that repels the particles and prevents them from aggregation.

Table 1. TEM imaging, absorbance study, shape tracing and size distribution of Gold nanoparticles

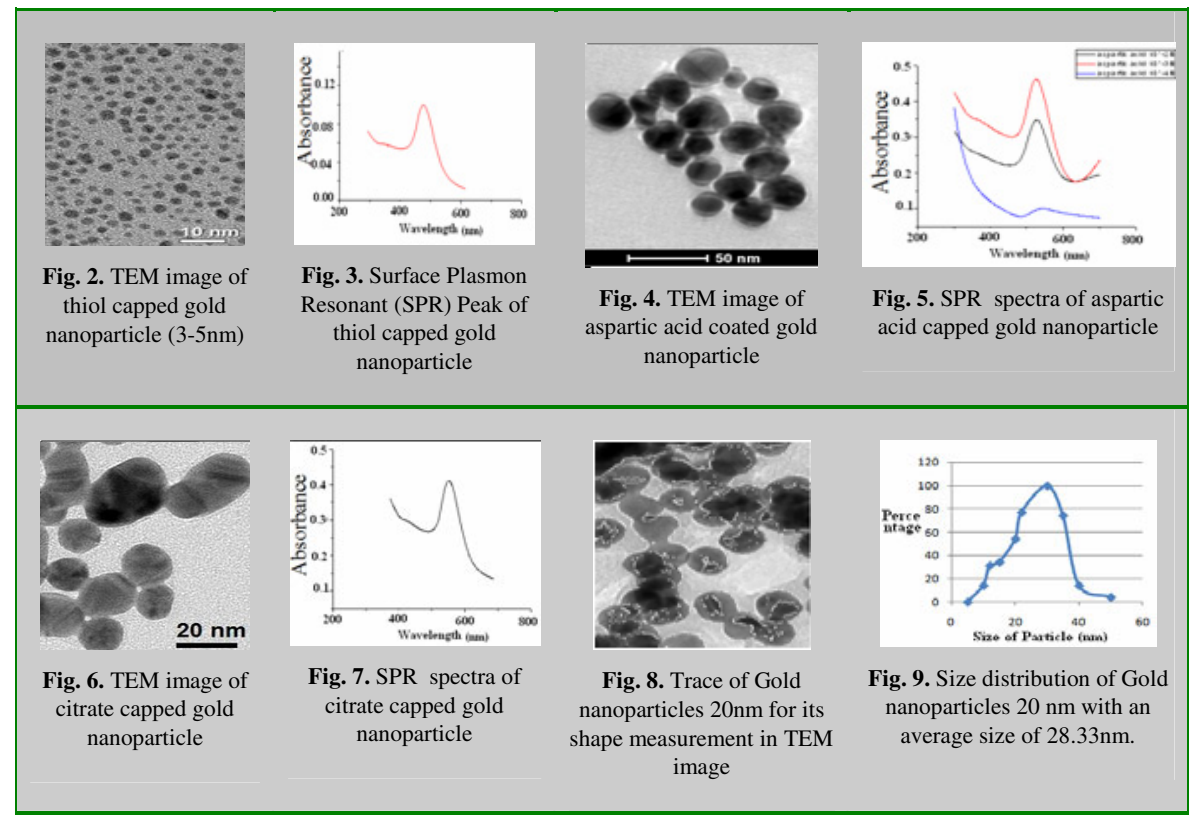


Table 2. Image Segmentation using Fuzzy C-Means

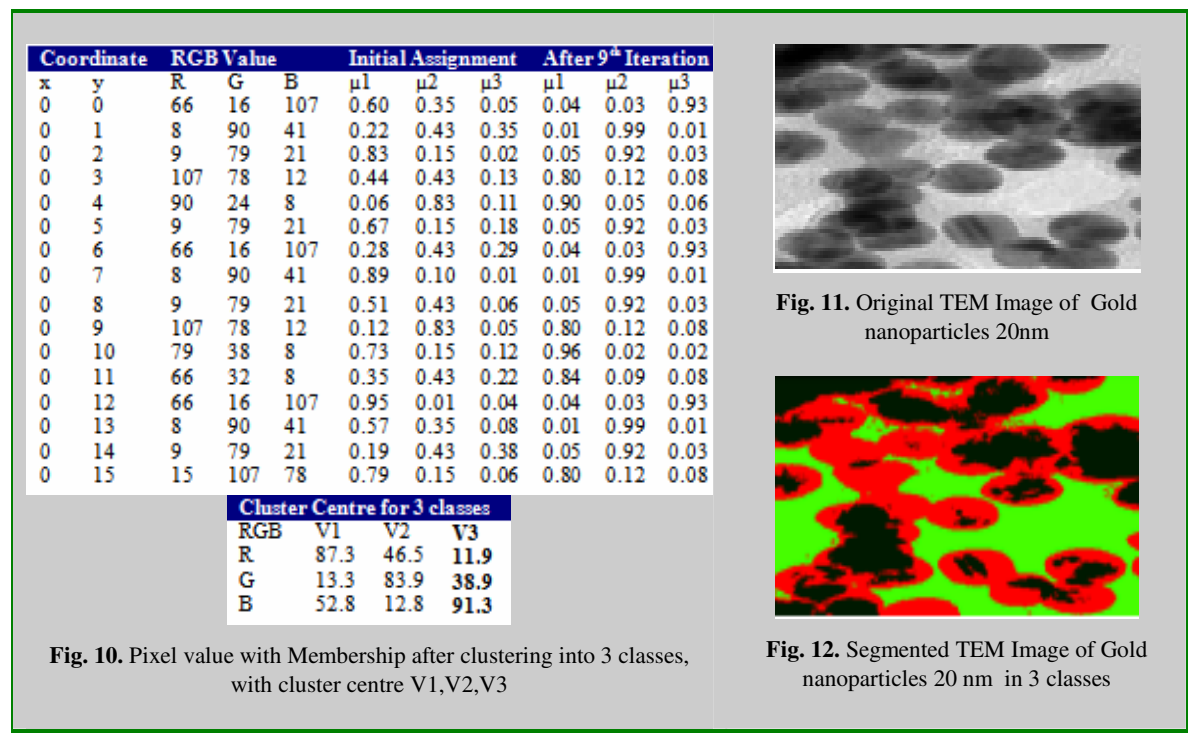

\section{Conclusion}

A novel and simple method for measurement of the size, shape and other spectral response of gold NPs. These values may be used as the input of Artificial Neural Networks(ANN) to characterized the synthesis process of gold NPs as proposed in fig.15. The ANN offers a successful tool for NPs preparation analysis and modeling. Chemical routes for the Polymer based nanocomposits preparation offer the advantage of (a) a cluster or atomic level control, and (b) an efficient scale up for processing and production. Here the size and shape of the nanoparticles are measured using fuzzy based clustering and shape analysis and gives better results than automatic threshold generator. The high degree of particle size and shape measurement in the $\mathrm{nm}$ range suggest a successful application of this method to conclude the signature of quantum size effects occurring in the gold nano particles, realization of color filters, UV absorbers with particularly interesting performances. The flexibility offered by the choice of different polymeric agents as a future element of interest towards future advances in nano-optical and nano-bio applications. 
Table 3. Packing Density and S/V Measurement of Gold NP's (2nm)

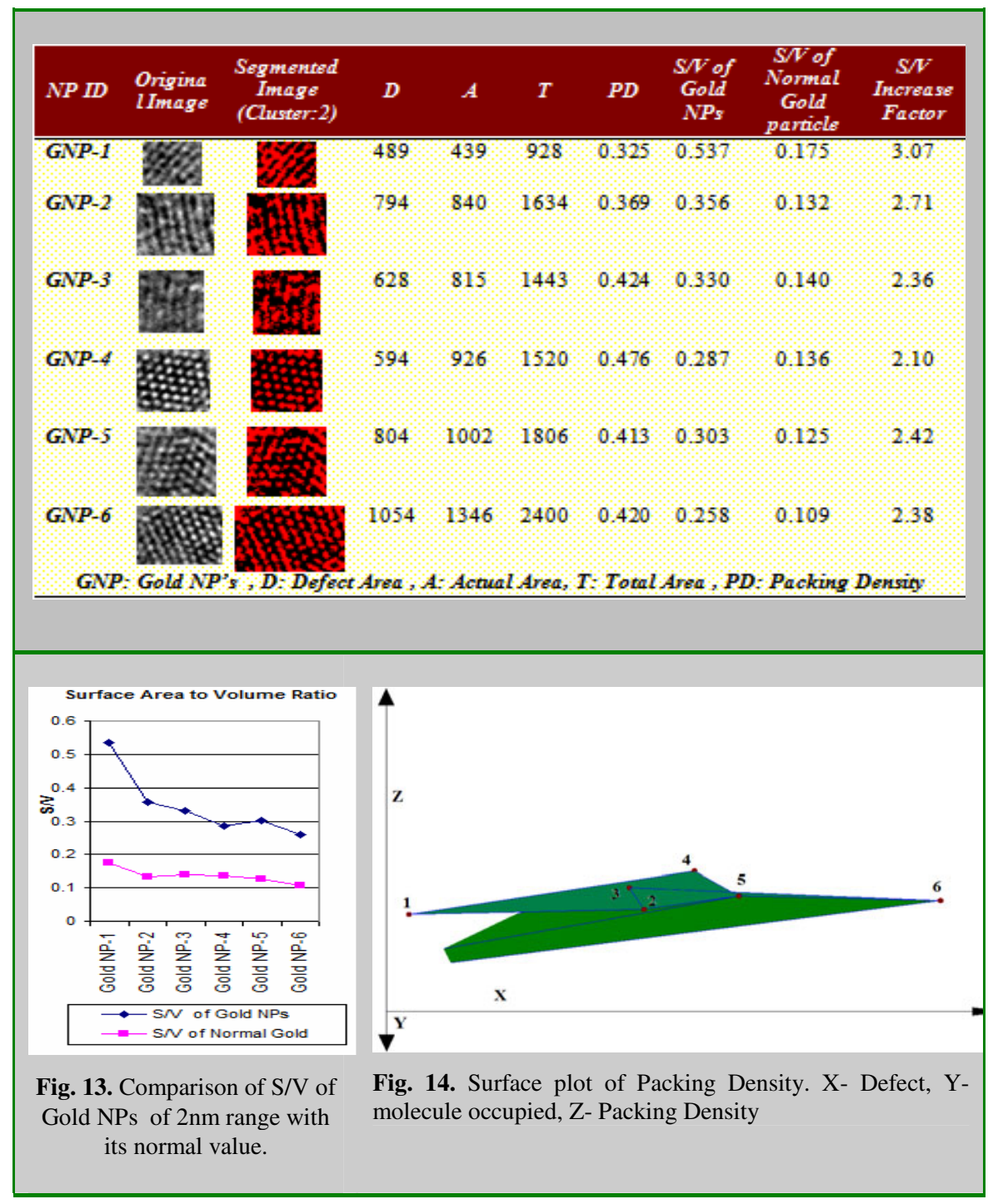




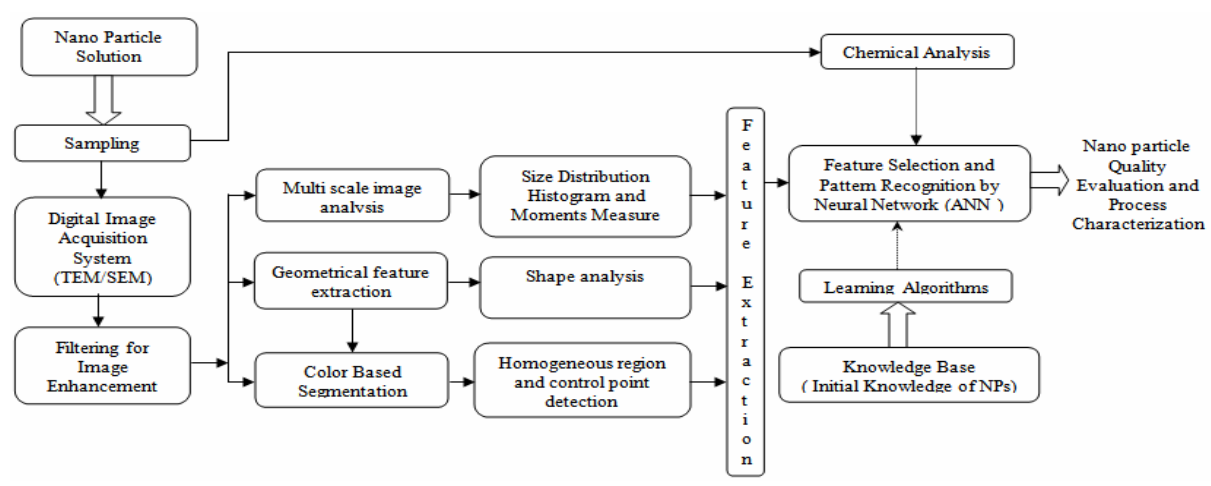

Fig. 15. Proposed architecture of NPs in process characterization

\section{Acknowledgement(s)}

We are thankful particularly to All engineers and Staff of Institute of Cybernetics Systems And Information Technology (ICSIT), all our colleagues at the Biological Science Division, Indian Statistical Institute, Kolkata and Department of Radiophysics and Electronics, Calcutta University, Kolkata.

\section{References}

1. Dutta Majumder, D., Karan, S., Goswami, A.: Characterization of Gold and Silver Nanoparticles using its Color Image Segmentation and Feature Extraction Using Fuzzy CMeans Clustering and Generalized Shape Theory. In: Proc. IEEE, International Conference on Communications and Signal Processing-2011, pp. 70-74 (2011)

2. Drexler, E.: Nano Systems: Molecular Machinery, Manufacturing, and Computation. MIT PhD thesis. Wiley, New York (1991)

3. Patra, P., Roy, I., Kumar, R., Gopal, M., Devakumar, C., Gogoi, R., Srivastava, C., Subramanium, B.S., Goswami, A.: Characterization of Nanocomposites in Flyash for Possible Pesticide Application. In: ICANN-2009, pp. 144-147. American Institute of Physics (2010)

4. González, R.C.: Richard Eugene Woods: Digital image processing. Prentice-Hall, Englewood Cliffs (2008)

5. Chanda, B., Dutta Majumder, D.: Digital Image Processing and analysis. PHI Learning Pvt. Ltd., New Delhi, India (2009)

6. Lindeberg, T.: Feature detection with automatic scale selection (abstract). International Journal of Computer Vision 30(2), 77-116 (1998)

7. Myllynen, P.: Nanotoxicology: Damaging DNA from a distance. Nature Nanotechnology 4, 795-796

8. Majumder, D., Banaerjee, R., Ulrichs, C., Mewis, I., Goswami, A.: Nano-Materials: Science of Bottom-Up and Top-Down, IETE Technical Review. Nanotechnology Education-a Paradigm Shift 24(1), 9-23 (2007)

9. Bezdek, J.C.: Pattern Recognition with Fuzzy Objective Function Algorithms. Plenum Press, New York and London 
10. Bezdek, J.C., Ehrlich, R., Full, W.: FCM: The fuzzy c-means clustering algorithm. Computers \& Geosciences 10(2-3), 191-203 (1984)

11. Bezdek, J.C., Hathaway, R.J., Sabin, M.J., Tucker, W.T.: Convergence theory for fuzzy Cmeans: counter examples and repairs. IEEE Trans. Syst. Man Cybern., 17873-17877 (1987)

12. Hathaway, R.J., Hu, Y.: Density-weighted fuzzy c-means clustering. IEEE Transactions on Fuzzy Systems 17(1), 243-252 (2009)

13. Parui, S., Sarma, E., Majumder, D.: How to discriminate shape using shape vector. Pattern Recognition Lett. 4, 201-204 (1986)

14. Dutta Majumder, D.: A Study on a Mathematical Theory of Shapes In Relation To Pattern Recognition And Computer Vision. India Journal of Theoretical Physics 43(4), 19-30 (1995) 